\title{
Blood Sampled Through Dried Blood Spots (DBS) Exhibits Diminished Ex vivo Metabolism Compared to Whole Blood Through Use of a Kinetic Isotope-Labeling Metabolomics Approach
}

\author{
Collin Hill' ${ }^{1}$, Jeremy Drolet ${ }^{1}$, Mark D Kellogg ${ }^{2}$, Vladimir Tolstikov ${ }^{1}$, Niven R Narain ${ }^{1}$ and Michael A Kiebish ${ }^{1 *}$
}

${ }^{1}$ Department of Precision Medicine, BERG, LLC, 500 Old Connecticut Path, Framingham, MA, 01701, USA

${ }^{2}$ Department of Laboratory Medicine and Pathology, Harvard Medical School, Boston Childrens' Hospital, Boston, MA, 02115, USA

\begin{abstract}
Blood is the primary matrix for metabolite profiling, providing a means for biomarker identification, pharmacokinetic/ pharmacodynamic analysis and disease monitoring. Conventional methodologies of blood sample collection require blood drawn by venous puncture. However, this technique allows for residual ex vivo metabolic activity of the blood matrix, thus presenting a challenge to capturing a physiologically representative readout of the metabolome. Blood that is not immediately processed is subjected to extended periods of ex vivo metabolism. Even when samples are transported by cold storage, some enzymatic processes remain active. The dried blood spot (DBS) collection technique renders cells metabolically inactive in a short span of time. We demonstrate that whole blood deposited onto a DBS card decreases uptake and metabolism of U13C-glucose after 4 hours, as analyzed by mass spectrometry. The cells also exhibit no further metabolic activity for up to 24 hours, whilst blood stored in a collection tube continue to actively uptake and metabolize U13C-glucose for up to 24 hours post-collection. Given that glycolysis is one of the most active pathways in blood cells, the ability to arrest glucose metabolism in a short amount of time is important to accurately capture the metabolite profile at the time of collection. We assert that this likely extends beyond glucose metabolism, as blood cells are capable of taking up other extracellular nutrients. We believe blood collection using the DBS technique offers an informative readout of the metabolome, compared to conventional blood collection, which is critical for population health and precision medicine applications.
\end{abstract}

Keywords: Dried blood spot; Metabolomics; Glucose metabolism; Mass spectrometry; Isotope-labeling

\section{Introduction}

Analysis of blood collected via deposition onto filter paper, also known as dried blood spot analysis (DBS) is primarily used in newborn screening to diagnose inborn errors of metabolism [1]. DBS is also utilized for drug monitoring [2,3], genomics [4], proteomics $[5,6]$ and metabolomics analysis $[7,8]$ in both clinical and pre-clinical settings. While the clinical applications are well-established, their use in pre-clinical studies has become increasingly popular in recent years, for example their utility in animal pharmacokinetic studies [9] Blood collected during clinical trials for PK/PD or blood parameter determination is often transported to offsite locations for processing and analysis, introducing pre-analytical sources of variability. From venous puncture to delivery of the sample at the analysis site, a substantial amount of time may pass and the sample matrix may undergo metabolic changes. The same challenges hold true in situations where clinical sites may not strictly follow protocols for handling and processing.

Studies have shown that blood left unprocessed and maintained at room temperature for as little as two hours post-collection is sufficient to alter metabolite readouts [10]. In particular, large increases in hypoxanthine and sphingosine-1-phosphate levels have been observed in whole blood samples that were left to coagulate at room temperature to be processed for separation of serum [10]. It is worth noting that 30 60 minutes is the recommended time to allow for clot formation and separation of serum [11], but sometimes there are extenuating logistical circumstances that prohibit immediate processing. Another study reported dramatic changes in glucose, pyruvate and lactate in blood samples when exposed to room temperature conditions for 24 hours [12]. The delay time in processing blood samples and the temperature at which samples are stored are most detrimental to the blood metabolome [13]. Steps such as maintaining collection tubes on ice can minimize the impact, but metabolism is only slowed during the 4-6 hour periods tested [13]. However, these studies did not investigate longer cold storage times. Interestingly, recent work by others have shown that metabolite profiles can detect delays in blood sample processing greater than 2 hours from the blood draw. In the study by Jain et al., a total of 149 of 803 metabolites changed during the 20 hour period in which samples were subjected to processing delays [14]. These data demonstrate that the unprocessed whole blood creates a skewed metabolic signature. Glucose, creatinine, acylcarnitines, urea, uric acid, ammonia, bilirubin, bile acids, cholesterol, amino acids and fatty acids [15] are examples of the types of metabolites that are often characterized in a clinical setting through blood tests that could possibly be affected by metabolic processes that remain active post-collection. In addition to the effects of temperature and processing time on blood metabolism, the venous blood drawing process subjects cells to extensive hemolysis which has been shown to have profound effects on blood metabolite profiles [10].

Given that cells exhibit active metabolism in the ex vivo setting, measuring metabolites in a matrix where metabolism is quickly quenched is highly desirable. In this study, we compared uptake and metabolism of uniformly $13 \mathrm{C}$ labeled (U13C) glucose in a tube against

*Corresponding author: Michael Kiebish, Chief Precision Medicine Officer, BERG, LLC, 500 Old Connecticut Path, Building B, Framingham, MA 01701 USA, Tel: (617) 588-2245; E-mail: Michael.Kiebish@BergHealth.com

Received: June 02, 2017; Accepted: June 26, 2017; Published June 29, 2017

Citation: Hill C, Drolet J, Kellogg MD, Tolstikov V, Narain NR, et al. (2017) Blood Sampled Through Dried Blood Spots (DBS) Exhibits Diminished Ex vivo Metabolism Compared to Whole Blood Through Use of a Kinetic Isotope-Labeling Metabolomics Approach. Biochem Anal Biochem 6: 325. doi: 10.4172/2161-1009.1000325

Copyright: () 2017 Hill C, et al. This is an open-access article distributed unde the terms of the Creative Commons Attribution License, which permits unrestricted use, distribution, and reproduction in any medium, provided the original author and source are credited. 
Citation: Hill C, Drolet J, Kellogg MD, Tolstikov V, Narain NR, et al. (2017) Blood Sampled Through Dried Blood Spots (DBS) Exhibits Diminished Ex vivo Metabolism Compared to Whole Blood Through Use of a Kinetic Isotope-Labeling Metabolomics Approach. Biochem Anal Biochem 6 : 325. doi: 10.4172/2161-1009.1000325

A

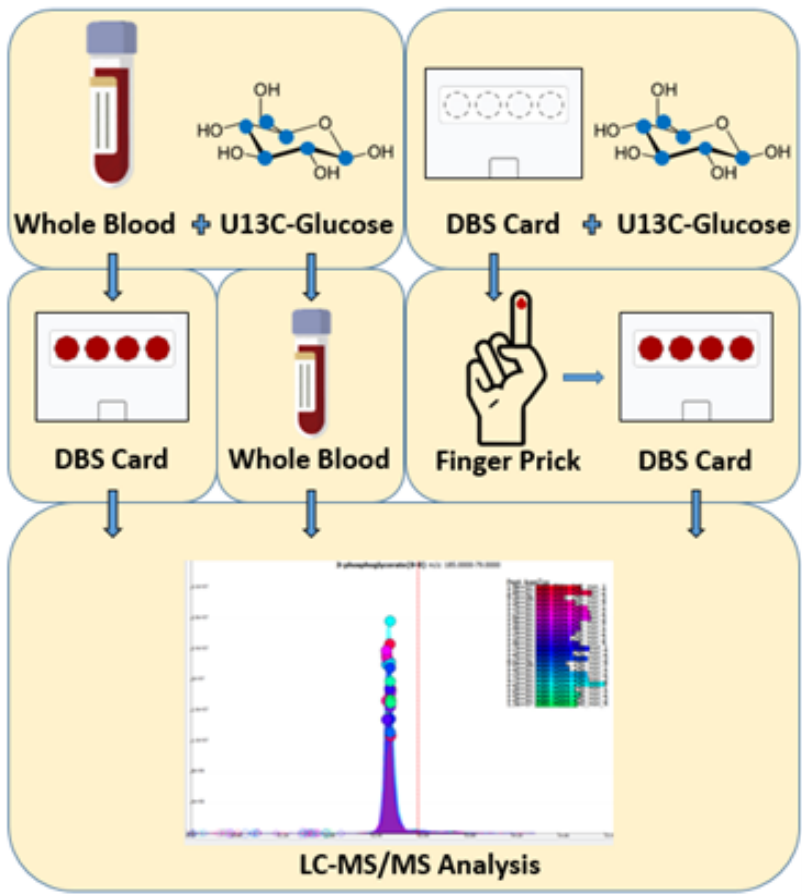

B

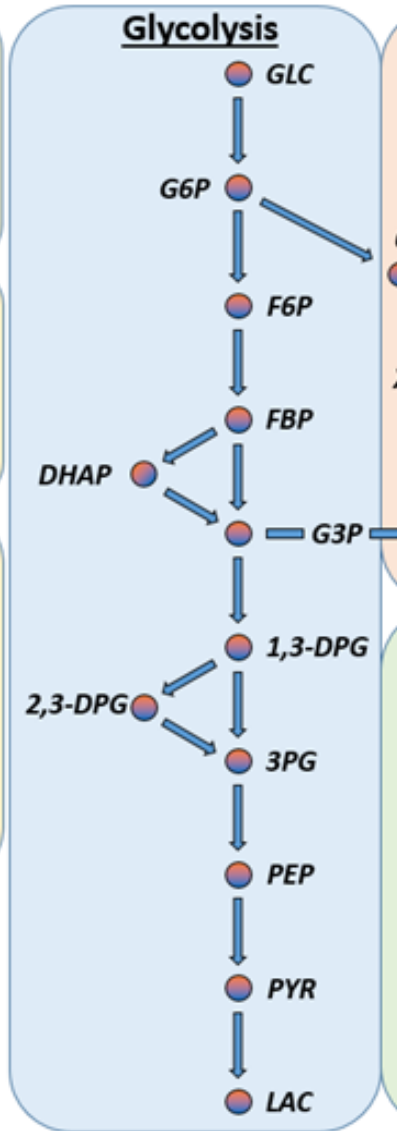

C

\section{Pentose Phosphate Pathway}

6PG

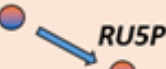
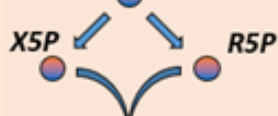

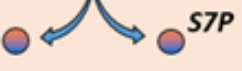

GLC $=$ Glucose

G6P $=$ Glucose-6-phosphate FGP $=$ Fructose-6-phosphate $\boldsymbol{F B P}=$ Fructose-1,6-Bisphosphate DHAP = Dihydroxyacetone phosphate $\mathbf{G} \mathbf{P} \boldsymbol{P}=$ Glyceraldehyde -3 -phosphate 1,3-DPG $=1,3-$ Disphosphoglycerate 2,3-DPG $=2,3$-Disphosphoglycerate 3PG $=3$-phosphoglycerate PEP $=$ Phosphoenolpyruvate PYR $=$ Pyruvate $L A C=$ Lactate

6PG = 6-phosphogluconate RUSP $=$ Ribulose -5 -phosphate $X 5 P=$ Xylulose-5-phosphate R5P $=$ Ribose-5-phosphate $S 7 \boldsymbol{P}=$ Sedoheptulose-7-phosphate

Figure 1: Schematic of blood collection protocol and glycolysis and pentose phosphate pathways. (A) Blood was collected in standard blood collection tubes and incubated with U13C-glucose for 4 hours. Liquid blood was either spotted onto a DBS card, or stored in liquid for 4 and 24 hours prior to quenching of metabolism and metabolomics analysis. Blood was also directly spotted onto a DBS card by fingerstick previously coated with U13C-glucose and allowed to dry before analysis. (B)

Glycolysis pathway and (C) pentose phosphate pathway metabolites whose isotopic enrichment was measured in blood samples.

blood sampled directly onto a DBS card containing dry U13C-glucose (Figure 1A). We also compared uptake and metabolism of U13Cglucose in a tube against blood transferred to a DBS card over a 24 hour period (Figure 1A). Other studies have used stable-isotope techniques in vivo to study blood cell metabolism [16] but as far as we are aware, this is the first study to compare ex vivo U13C-glucose metabolism on a DBS card with traditional blood collection techniques.

\section{Methods}

\section{Materials}

U13C-glucose was obtained from Cambridge Isotope Laboratories, Inc. (Andover, MA). Tributylamine and acetic acid were obtained from Sigma-Aldrich (St. Louis, MO). Whatman 903 protein saver DBS cards, LC-MS grade water, LC-MS grade Methanol and DPBS were obtained from Fisher Scientific (Pittsburgh, PA). K2-EDTA-coated tubes and alcohol prep swabs were obtained from Beckton-Dickinson, BD (Franklin Lakes, NJ). Accu-chek lancets were obtained from Roche (Basel, Switzerland).

\section{Preparation and collection of samples}

Written informed consent was provided by the subjects prior to participating in the study. Research use of samples was conducted in accordance with the terms outlined within the informed consent form and the terms set forth therein and with the tenets of the Declaration of Helsinki. Diets and other physiologic parameters were not controlled in this study. A total of 6 healthy volunteers ( 3 male and 3 female) were sampled (mean age $30 \pm 7$ ).

A stock solution of U13C-glucose (100X) was made up at $500 \mathrm{mM}$

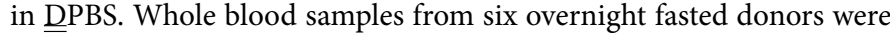
collected by venipuncture using a $21 \mathrm{~g}$ butterfly needle into K2-EDTAcoated tubes and immediately transferred to a $5 \mathrm{~mL}$ non-coated tube containing the $100 \mathrm{X}$ stock of U13C-glucose, for a final concentration of $5 \mathrm{mM}(\mathrm{n}=3) .50 \mu \mathrm{L}$ of the U13C-glucose spiked blood was transferred by pipette to create one spot each on two separate DBS cards and left to dry for 4 and 24 hours. For whole blood samples left in the coated tube, at 4 and 24 hours, $100 \mu \mathrm{L}$ was transferred to a fresh tube. Metabolism was quenched by snap freezing the tube in liquid nitrogen for 5 minutes. The whole blood samples were stored at $-80^{\circ} \mathrm{C}$ for a week until analysis.

For samples that were collected by self-administered fingerstick, DBS cards were first pre-treated with $25 \mu \mathrm{L}$ of a 1X-U13C-glucose stock in DPBS to yield a final concentration of $5 \mathrm{mM}$ after addition of blood. The cards were then allowed to dry overnight at room temperature. Donors prepped their fingers by first disinfecting with an alcohol wipe, letting it dry and then sampled blood with a lancet, spotting enough to 
Citation: Hill C, Drolet J, Kellogg MD, Tolstikov V, Narain NR, et al. (2017) Blood Sampled Through Dried Blood Spots (DBS) Exhibits Diminished Ex vivo Metabolism Compared to Whole Blood Through Use of a Kinetic Isotope-Labeling Metabolomics Approach. Biochem Anal Biochem 6 : 325. doi: 10.4172/2161-1009.1000325

cover each of the four spots on the card. The blood spots were left to dry for four hours at room temperature. Due to the small volume of blood collected from a single fingerstick, only one card of four blood spots was collected per donor via fingerstick.

Blood from DBS cards was extracted either at 4 or 24 hours. A 6-millimeter spot was cored using a manual puncher (GE Healthcare) and transferred to a tube containing $300 \mu \mathrm{L} 80 \%$ ice cold methanol. The puncher was rinsed with $50 \%$ methanol and dried in between cards to prevent cross-contamination. The spots in $80 \%$ cold methanol were then sonicated for 10 minutes. Whole blood samples for all time points were extracted at 24 hours post collection as follows: Whole blood was thawed at room temperature for 10 minutes. $100 \mu \mathrm{L}$ of whole blood was aliquoted into an Eppendorf tube and $900 \mu \mathrm{L} 80 \%$ ice cold methanol was added. The tubes were sonicated for 10 minutes. Both DBS and whole blood samples were then centrifuged at $14,000 \mathrm{rpm}$ for 10 minutes at $4^{\circ} \mathrm{C}$. The entire $300 \mu \mathrm{L}$ DBS lysate and $100 \mu \mathrm{L}$ whole blood supernatant was transferred to a 96 well plate and evaporated to dryness overnight in a centrifugal evaporator (Labconco, Kansas City, $\mathrm{MO}$ ). Dried DBS lysate was stored at $-80 \mathrm{C}$ for a week until analysis. Samples were removed from the freezer, reconstituted in $50 \mu \mathrm{L} \mathrm{LC}$ MS grade water and spun to remove excess debris at $14,000 \mathrm{rpm}$ for 10 minutes at $4^{\circ} \mathrm{C}$ just prior to analysis.

\section{Liquid Chromatography Mass Spectrometry (LC-MS/MS) analyses of samples}

Glucose metabolites and their isotopically labeled forms were quantified using an LC-MS technique adapted from a method previously described by $\mathrm{Lu}$ et al. [17]. Briefly, $10 \mu \mathrm{L}$ of sample was injected and separated using a Waters Acquity UPLC over a 25-minute tributylamine ion-pair/methanol gradient. A Thermo TSQ Vantage triple-quadrupole mass-spectrometer was operated in negative mode, collecting SRM (selected reaction monitoring) for the metabolites of interest and their isotopically labeled forms (Supplementary Table 1). The following values were used to operate the mass spectrometer: spray voltage, $-3250 \mathrm{~V}$, vaporizer temperature, $300^{\circ} \mathrm{C}$, capillary temperature, $360^{\circ} \mathrm{C}$, auxiliary gas, $15 \mathrm{AU}$, sheath gas, $32 \mathrm{AU}$, sweep gas, $0.5 \mathrm{AU}$, collision gas, $1.5 \mathrm{~m}$ Tor, dwell time $30 \mathrm{~ms}$.

\section{Mass spectrometry data processing}

Data was processed by first converting the files into the common
mzML format using the MSConvert tool [18]. Peak areas for metabolites and their isotopically labeled forms were integrated using the freeware MAVEN metabolomics data processing tool [19]. Postprocessing calculations were done using in-house scripts through R-Studio. Correction for the natural abundance of $\mathrm{C}^{13}$ was done first, followed by calculation of isotope enrichment and total metabolite pool sizes. Fraction isotope enrichment calculations were done by dividing each isotopologue by the summation of all isotopologues for a given metabolite, including both labeled and unlabeled forms. Pool size calculations were carried out by summing all data for all isotopologues, both labeled and unlabeled forms.

For isotope-enrichment data shown in heat maps, the average of all replicates for the fully-labeled isotopologue within each donor is shown. For pool size data, first the replicates for each donor were averaged at the specified time points. Fold-change was calculated by dividing the 24 hour pool size by the 4 hour pool size for each donor. The foldchanges at each time point were then averaged. Standard deviations of the fold-change values were used to calculate and plot the associated errors of measurement.

\section{Statistical analysis}

All data was analyzed by GraphPad Prism software. Data displayed in heat maps are the average of fraction enrichment replicates for each of the six donors at the relevant time points. Two-tailed T-tests were used for pairwise analysis. Appropriate post-hoc tests were performed as detailed in the figure legends.

\section{Results}

Isotopic enrichment of glycolytic and pentose phosphate pathway intermediates in blood sampled directly onto a DBS card was lower when compared to conventional blood collection

A comparison of metabolites enriched by U13C-glucose in liquid whole blood and spotted onto a DBS card (WB to DBS) versus fingerstick deposited DBS blood samples followed by 4 hours of drying (DBS) is detailed in Figure 2. Whole blood that was transferred to a DBS card showed a marked decrease in the fraction enrichment of downstream metabolites of glucose, when compared to whole blood left in a tube.

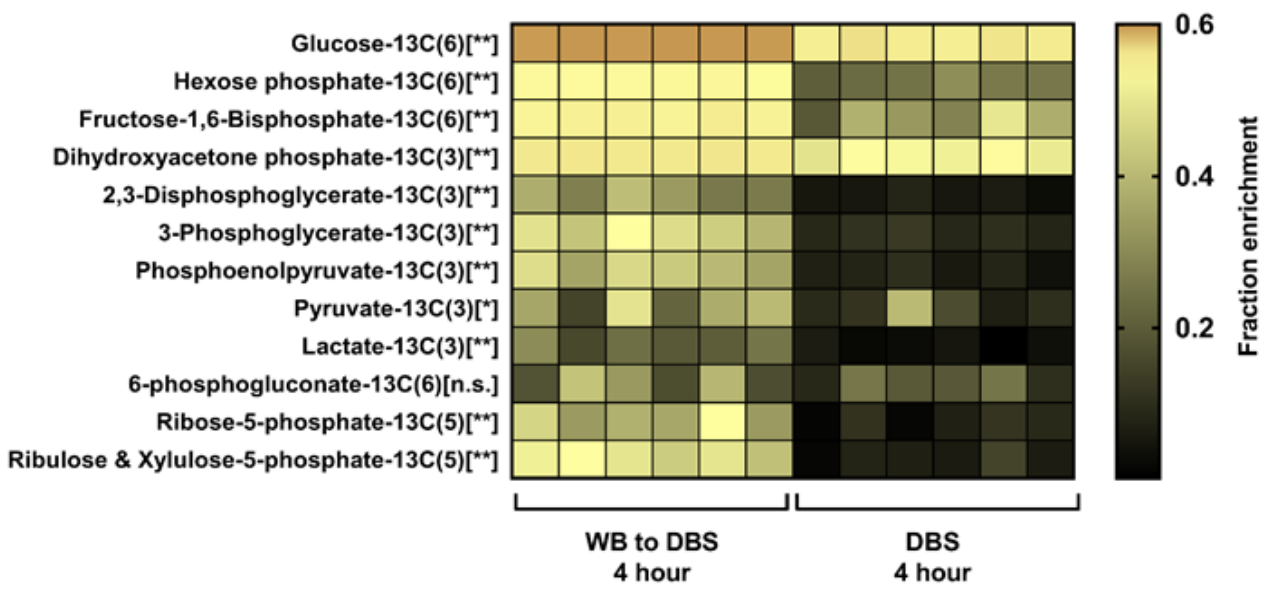

Figure 2: Heat map of glycolysis and pentose phosphate pathway metabolite enrichment by U13C-glucose after 4 hours, in whole blood to DBS, and blood collected directly onto a DBS card. Whole blood to DBS (WB to DBS) samples showed enrichment of glycolysis and pentose phosphate metabolites, while blood spotted directly onto a DBS card (DBS) showed much less enrichment after 4 hours. Data is shown as average of replicates for each individual donor and analyzed by multiple T-tests comparing each metabolite followed by the false discovery rate test and two stage step up method of Benjamini, Krieger and Yekutieli. ( ${ }^{*} p<0.05$, $\left.{ }^{* *} p<0.0001\right)$. 
Citation: Hill C, Drolet J, Kellogg MD, Tolstikov V, Narain NR, et al. (2017) Blood Sampled Through Dried Blood Spots (DBS) Exhibits Diminished Ex vivo Metabolism Compared to Whole Blood Through Use of a Kinetic Isotope-Labeling Metabolomics Approach. Biochem Anal Biochem 6 : 325. doi: 10.4172/2161-1009.1000325

Page 4 of 6

\begin{tabular}{|c|c|c|c|c|}
\hline Metabolite & WB vs. WB to DBS 4 hours & WB vs. WB to DBS 24 hours & WB 4 vs. 24 hours & WB to DBS 4 vs. 24 hours \\
\hline Glucose-13C (6) & 0.47 & 0.36 & 0.85 & 0.82 \\
\hline Hexose-phosphate-13C (6) & 1.63E-07 & $4.55 \mathrm{E}-12$ & 2.11E-09 & 0.74 \\
\hline Fructose-1,6-Bisphosphate-13C (6) & $1.02 \mathrm{E}-05$ & 9.74E-09 & $5.81 \mathrm{E}-08$ & 0.94 \\
\hline Dihydroxyacetone-phosphate-13C (3) & $9.28 \mathrm{E}-04$ & $7.72 \mathrm{E}-11$ & 4.71E-09 & 0.65 \\
\hline 2,3-Diphosphoglycerate-13C (3) & $1.51 \mathrm{E}-07$ & $1.20 \mathrm{E}-14$ & 7.17E-12 & 0.14 \\
\hline 3-Phosphoglycerate-13C (3) & 3.68E-07 & $3.00 \mathrm{E}-15$ & $5.78 \mathrm{E}-12$ & 0.13 \\
\hline Phosphoenolpyruvate-13C (3) & $1.14 \mathrm{E}-06$ & $4.30 \mathrm{E}-14$ & $1.20 \mathrm{E}-10$ & 0.25 \\
\hline Pyruvate-13C (3) & 7.53E-07 & $5.44 \mathrm{E}-10$ & $2.26 \mathrm{E}-08$ & 0.27 \\
\hline Lactate-13C (3) & $1.88 \mathrm{E}-11$ & $1.40 \mathrm{E}-14$ & $8.41 \mathrm{E}-12$ & 0.18 \\
\hline 6-phosphogluconate-13C (6) & $3.41 \mathrm{E}-04$ & $1.64 \mathrm{E}-07$ & 6.35E-05 & 0.69 \\
\hline Ribose-5-phosphate-13C (5) & 1.16E-08 & $6.45 \mathrm{E}-10$ & $1.68 \mathrm{E}-04$ & 0.65 \\
\hline Ribulose \& Xylulose-5-phosphate-13C (5) & $2.64 \mathrm{E}-11$ & 2.58E-09 & 1.20E-03 & 0.49 \\
\hline
\end{tabular}

Table 1: $p$-values for pairwise comparison of isotopic enrichment across multiple time points, depicted in Figure 3

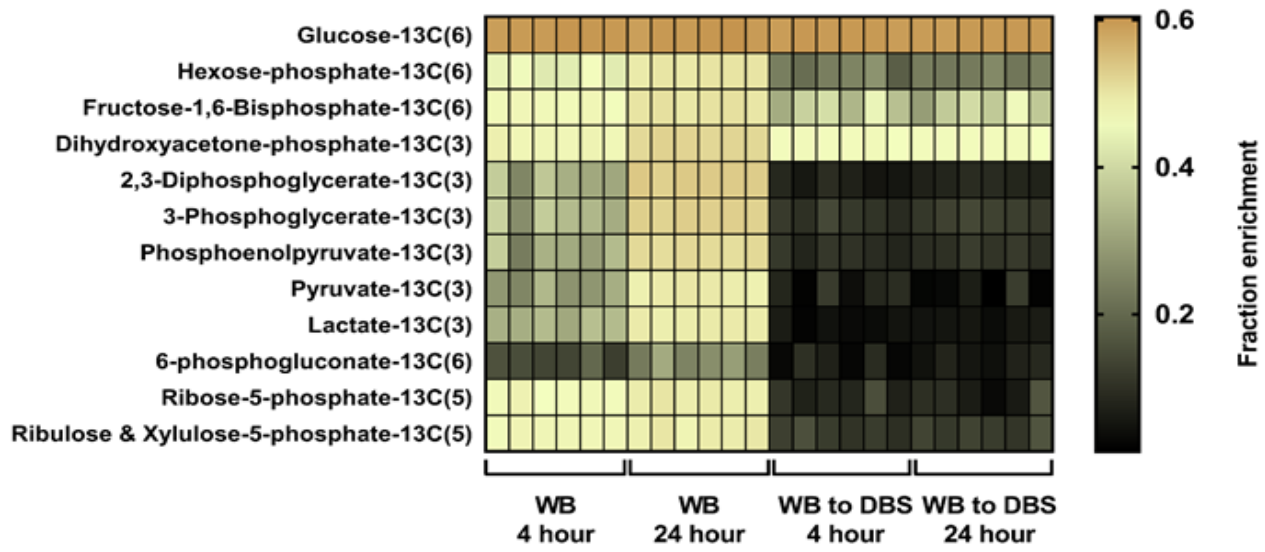

Figure 3: Heat map of glycolysis and pentose phosphate pathway metabolites enriched by U13C-glucose in whole blood or whole blood spotted onto a DBS card and dried for 4 and 24 hours. (A) Whole blood samples (WB) at both 4 and 24 hours show much more enrichment of glycolysis and pentose phosphate metabolites compared with blood spotted directly onto a DBS card (WB to DBS) at 4 and 24 hours. Blood spotted onto a DBS card shows no further enrichment when comparing 4 and 24 hour values. Data is shown as average of replicates for each individual donor and analyzed by multiple T-test comparing each metabolite followed by the false discovery rate test and two stage step up method of Benjamini, Krieger and Yekutieli.

\section{Whole blood spotted onto a DBS card arrests glucose metab- olism compared with conventional blood collection}

We then compared the uptake and metabolism of U13C-glucose in whole blood stored for 4 and 24 hours at room temperature in a tube prior to quenching of metabolism (WB) with liquid whole blood that was incubated with U13C-glucose and transferred to a DBS card and subsequently dried (WB to DBS). A heat map demonstrating lower enrichment of glucose metabolites in WB to DBS versus WB, as well as the p-values for each time point comparison is detailed in Figure 3 and Table 1 . Whole blood that was stored in a tube for 4 hours was significantly more enriched for glycolytic and pentose phosphate pathway intermediates when compared with whole blood spotted onto a DBS card (Figure 3, WB 4 hours compared with WB to DBS 4 hours, $\mathrm{p}<0.001)$. Comparison between whole blood stored for 4 hours and 24 hours demonstrated progressive enrichment of glycolytic pathway metabolites at 24 hours (Figure 3, WB 4 hours compared with WB 24 hours, $\mathrm{p}<0.01$ ), suggesting that prolonged storage of blood in liquid form at room temperature allows metabolic processes to continue. Furthermore, comparing 4 hours with the 24 hours drying times of the DBS samples, there is no further enrichment of glucose metabolites (Figure 3, WB to DBS 4 hours compared with WB to DBS 24 hours, $\mathrm{p}>0.1$ ). Given this information, we affirm that during the drying time on the card, blood cells begin to slow ex vivo glucose metabolism.
Pyruvate, lactate and ATP accumulate in whole blood stored in liquid form

Lactate is the end product of glucose metabolism in the erythrocyte due to their lack of mitochondria. In their physiological environment, erythrocytes excrete about $20 \%$ of the lactate delivered and taken up daily to the liver and kidneys and converted to glucose as part of the Cori Cycle [20]. Therefore, production and accumulation of the excretion product lactate is indicative of a metabolically active blood cell. In this study, comparing 4 hour against 24 hour pool sizes, there is approximately a 3 -fold increase in total pyruvate and a 4 -fold increase in the total amount of lactate produced in stored whole blood (WB) compared to blood spotted onto a DBS card (WB to DBS), both from labeled and unlabeled glucose sources. (Figure $4 \mathrm{~A}, \mathrm{p}<0.0001$ ). We also demonstrate that whole blood shows a statistically significant $16 \%$ increase in their ATP levels over a 24 hour period when compared to the 4 hour time point (Figure $4 \mathrm{~B}, \mathrm{p}<0.05$ ), while ATP levels remain consistent in blood cells left to dry on a DBS card. This observation of increased ATP is consistent with increased activity of the ATPproducing enzyme lactate dehydrogenase, which also forms lactate. Of note, this is one of the two active ATP-producing enzymes that are active in the mature erythrocyte.

\section{Discussion}

Herein, we utilized an isotope labeling metabolomics approach to 
A

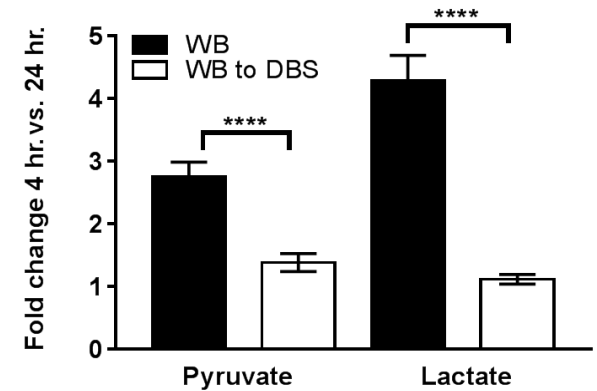

B

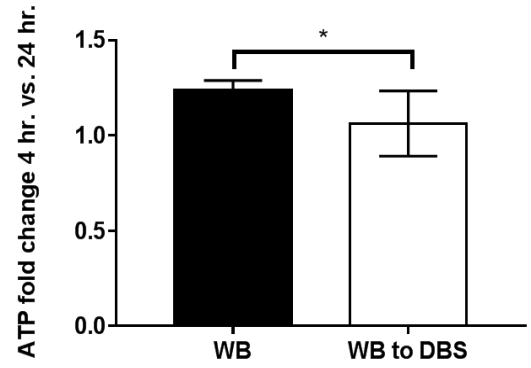

Figure 4: Fold change values of pyruvate, lactate and ATP pool sizes of stored whole blood and whole blood spotted on a DBS card, comparing 4 and 24 hour time points. (A) Pyruvate and lactate pool sizes are significantly higher in stored liquid whole blood (WB) than in blood spotted onto a DBS card (WB to DBS) when comparing 4 and 24 hour time points. Data is pooled from six individual donors and analyzed by two-tailed T-test with Welch's correction ( ${ }^{* * * *}<<0.0001$ ). (B) ATP levels are higher in stored liquid whole blood than blood spotted onto a DBS card when comparing 4 and 24 hour time points. Data is pooled from six individual donors and analyzed by two-tailed T-test with Welch's correction ( $\left.{ }^{*} p<0.05\right)$.

determine de novo synthesis of glucose metabolites by supplementing an isotopically labeled form in the ex vivo setting. By forming heavy metabolic products from the supplied heavy substrates, we were able to assess the metabolic activity of blood cells once removed from the body. This approach allows for precise determination of kinetic metabolic rates, whereas steady-state experiments only allow for static measurements of metabolite pool sizes. It also provides information on many enzymatic activities and isotope-enrichment was shown to be highly reproducible in whole blood, across all donors tested, regardless of gender (Supplementary Figure 1). With this information, we investigated whether DBS exhibited differential enrichment of glycolytic (Figure 1B) and pentose phosphate pathway (Figure 1C) intermediates by U13C-glucose when compared with whole blood, to determine if DBS offered a better means for characterizing the metabolome. Active metabolism in stored samples may alter the analyzed blood metabolome and provide inaccurate information of the physiologically relevant profile.

We observed that blood sampled by finger prick demonstrated decreased enrichment of the U13C-glucose itself when compared to whole blood, despite being treated with the same $5 \mathrm{mM}$ final concentration. This is possibly due to the directly spotted blood beginning to dry immediately preventing the glucose from entering into solution. The inability for glucose to be incorporated into solution accounts for the subsequent observation that DBS samples demonstrate lower enrichment of glucose metabolites when compared to WB samples, where glucose is readily solubilized and incorporated into its downstream products.

Conventionally collected blood demonstrated active metabolism of U13C-glucose into the downstream heavy products and metabolite pool sizes dramatically changed over a 24 hour period. As evidenced here, the DBS technique overcomes some of the challenges associated with obtaining accurate readouts from whole blood stored in a tube, as blood cell glucose metabolism is arrested in as little as the four hour drying time and extends to at least 24 hours later. Four hours was the earliest time point tested on the DBS card, as the blood takes several hours to be fully dry [21]. No further isotopically labeled products were produced from U13C-glucose after drying and total pool sizes of metabolites remain constant. Given the decrease in enrichment on DBS cards during this four hour period when compared to whole blood, it is evident that as the blood sample dries on the card the ability of the cells to utilize glucose is greatly diminished. Additionally, the lack of change in metabolite pool sizes measured also demonstrates the room temperature stability of the metabolites measured throughout a full day of drying on a DBS card.
Previous studies done in liquid whole blood report staggering alterations in glycolytic flow, especially accumulation of lactate when erythrocytes are removed from circulation [22]. The accumulating lactate and subsequent perturbation of the lactate/pyruvate ratio disturbs the NAD/NADH ratio and can further disturb activity of metabolic enzymes upstream. The glyceraldehyde-3-phosphate dehydrogenase (3-PGAD) enzyme of glycolysis is directly affected by the NAD/ NADH ratio and allows metabolite upstream to accumulate [22]. Our data shows that the DBS technique is able to prevent accumulation of pyruvate and lactate, while whole blood shows significant accumulation of these excretion products over a 24-hour-period, indicating persistent enzymatic activity. Aside from intracellular activities, it is important to note that the extracellular plasma matrix contains active enzymes which can catalyze metabolic reactions, such as acetylcholinesterase, glutamate-pyruvate transaminase and lactate dehydrogenase [23]. Studies have shown that certain enzymes are stable/catalytically active, even in situations of cold storage [23]. In particular, the previous study reports that there are changes in the activity of lactate dehydrogenase and alkaline phosphatase in whole blood under cold storage after just one day. In addition, they also report decreasing sodium levels and increasing potassium levels after one day of cold storage. Therefore, the whole blood matrix is active in several aspects, including ion flux and both intracellular/extracellular metabolic activities.

Since the blood matrix is comprised of $99 \%$ red blood cells and only $1 \%$ white blood cells [24], we attribute the majority of glucose metabolism to erythrocytes. While the active metabolic pathways of the erythrocyte are relatively few in number when compared to other cell types due to a lack of mitochondria, they are capable of taking up other nutrients as well. Besides glucose, erythrocytes maintain active adenine salvage pathways, as they are unable to produce ATP de novo. Adenine, hypoxanthine, and $S$-adenosylmethonine (SAM) can all provide the purine backbone for nucleotide salvage, whether it be directly or by formation as a metabolic product [25]. Furthermore, erythrocytes rely on the uptake of fatty acids for membrane repair, again due to their inability for de novo synthesis [26]. This is especially true in situations of oxidative stress, as reactive oxygen species (ROS) contribute to the degradation and integrity of the erythrocyte membrane. Although the dynamics of uptake of these nutrients were not investigated in this study, we can infer that since the extremely active glucose metabolic pathway is quenched through the DBS technique, other metabolic pathways will follow suit.

\section{Conclusion}

Whole blood stored in liquid form is subject to residual metabolic 
Citation: Hill C, Drolet J, Kellogg MD, Tolstikov V, Narain NR, et al. (2017) Blood Sampled Through Dried Blood Spots (DBS) Exhibits Diminished Ex vivo Metabolism Compared to Whole Blood Through Use of a Kinetic Isotope-Labeling Metabolomics Approach. Biochem Anal Biochem 6 : 325. doi: 10.4172/2161-1009.1000325

enzymatic activity in the ex vivo setting, and therefore may lead to inaccuracies in metabolite profiles. Herein, we assert that collection of blood through the DBS technique provides advantages over liquid blood, especially when investigating metabolic processes. Our data suggests that challenges associated with blood collection and subsequent metabolite profiling can be mitigated through the use of DBS as a collection and storage technique. Furthermore, it is noninvasive, relatively inexpensive, can be self-collected, and uses smaller volumes of blood compared to venipuncture. Use of DBS also simplifies transportation of samples, which can benefit both pre-clinical and clinical studies. Careful consideration of the analytes of interest, their stability, and any residual enzymatic activity that may affect their measurement is crucial when selecting a sample collection and storage process. The DBS technique can be widely applied and may be useful to prevent enzymatic activities when assessing biomarker identification, pharmacodynamic analysis, disease monitoring and precision medicine studies. However, one of the disadvantages of the DBS technique is the inability to fractionate the blood sample into plasma or serum and requires that whole blood is the matrix of interest.

\section{Acknowledgements}

The authors would like to thank K. Wilkinson for scientific writing assistance.

\section{Conflict of Interest}

This study was funded in its entirety by BERG, LLC. All authors are current or former employees of BERG, LLC and have stock options. NN is a co-founder of BERG, LLC.

\section{References}

1. Lehmann S, Delaby C, Vialaret J, Ducos J, Hirtz C (2013) Current and future use of "dried blood spot" analyses in clinical chemistry. Clin Chem Lab Med 51: 1897-1909.

2. Lauer E, Widmer C, Versace F, Staub C, Mangin P, et al. (2013) Body fluid and tissue analysis using filter paper sampling support prior to LC-MS/MS: application to fatal overdose with colchicine. Drug Test Anal 5: 763-772.

3. Li W, Tse FL (2010) Dried blood spot sampling in combination with LC-MS/MS for quantitative analysis of small molecules. Biomed Chromatogr 24: 49-65.

4. Tarini BA, Goldenberg AJ (2012) Ethical issues with newborn screening in the genomics era. Annu Rev Genomics Hum Genet 13:381-393.

5. Chambers AG, Percy AJ, Yang J, Borchers CH (2015) Multiple reaction monitoring enables precise quantification of 97 proteins in dried blood spots. Mol Cell Proteomics 14: 3094-3104.

6. Edwards RL, Griffiths P, Bunch J, Cooper HJ (2012) Top-down proteomics and direct surface sampling of neonatal dried blood spots: Diagnosis of unknown hemoglobin variants. J Am Soc Mass Spectrom 23: 1921-1930.

7. Wilson I (2011) Global metabolic profiling (metabonomics/metabolomics) using dried blood spots: advantages and pitfalls. Bioanalysis 3: 2255-2257.

8. Gao F, McDaniel J, Chen EY, Rockwell HE, Drolet J, et al. (2017) Dynamic and temporal assessment of human dried blood spot MS/MSALL shotgun lipidomics analysis. Nutr Metab (Lond) 14:28

9. Uyeda C, Pham R, Fide S, Henne K, Xu G, et al. (2011) Application of automated dried blood spot sampling and LC-MS/MS for pharmacokinetic studies of AMG 517 in rats. Bioanalysis 3: 2349-2356.
10. Yin P, Peter A, Franken H, Zhao X, Neukamm SS, et al. (2013) Preanalytical aspects and sample quality assessment in metabolomics studies of human blood. Clin Chem 59: 833-845.

11. Tuck MK, Chan DW, Chia D, Godwin AK, Grizzle WE, et al. (2009) Standard operating procedures for serum and plasma collection: Early Detection Research Network Consensus Statement Standard Operating Procedure Integration Working Group. J Proteome Res 8: 113-117.

12. Bernini P, Bertini I, Luchinat C, Nincheri P, Staderini S, et al. (2011) Standard operating procedures for pre-analytical handling of blood and urine for metabolomic studies and biobanks. J Biomol NMR 49: 231-243.

13. Jobard E, Tredan O, Postoly D, Andre F, Martin AL, et al. (2016) A systematic evaluation of blood serum and plasma pre-analytics for metabolomics cohor studies. Int J Mol Scis 17: 2035.

14. Jain M, Kennedy AD, Elsea SH, Miller MJ (2017) Analytes related to erythrocyte metabolism are reliable biomarkers for preanalytical error due to delayed plasma processing in metabolomics studies. Cli Chim Acta 466: 105-111.

15. Yin P, Lehmann R, Xu G (2015) Effects of pre-analytical processes on blood samples used in metabolomics studies. Analytical and bioanalytical chemistry 407: 4879-4892.

16. Coelho M, Mendes VM, Lima IS, Martins FO, Fernandes AB, et al. (2016) Direct analysis of $\left[6,6-(2) \mathrm{H}_{2}\right]$ glucose and [U-(13)C6] glucose dry blood spo enrichments by LC-MS/MS. J Chromatogr B Analyt Technol Biomed Life Sci 1022: $242-248$

17. Lu W, Clasquin MF, Melamud E, Amador-Noguez D, Caudy AA, et al. (2010) Metabolomic analysis via reversed-phase ion-pairing liquid chromatography coupled to a stand alone orbitrap mass spectrometer. Analytical chemistry 82 3212-3221.

18. Adusumilli R, Mallick P (2017) Data conversion with ProteoWizard MSconvert. Methods Mol Biol 1550: 339-368.

19. Clasquin MF, Melamud E, Rabinowitz JD (2012) LC-MS data processing with MAVEN: a metabolomic analysis and visualization engine. Curr Protoc Bioinformatics Chapter 14: Unit 14-11.

20. Park R (1980) Lactic acidosis. West J Med 133: 418-424.

21. Zakaria R, Allen KJ, Koplin JJ, Roche P, Greaves RF (2016) Advantages and challenges of dried blood spot analysis by mass spectrometry across the total testing process. Ejifcc 27: 288-317

22. Tilton WM, Seaman C, Carriero D, Piomelli S (1991) Regulation of glycolysis in the erythrocyte: The role of the lactate/pyruvate and NAD/NADH ratios. J Lab Clin Med 118: 146-152.

23. Heins M, Heil W, Withold W (1995) Storage of serum or whole blood samples? Effects of time and temperature on 22 serum analytes. European journal of clinical chemistry and clinical biochemistry. Eur J Clin Chem Clin Biochem 33: 231-238.

24. Diem KLC, Seldrup J (1975) Documenta Geigy Scientific Tables In: Lentne C (ed) Giegy Scientific Tables. Ciba-Giegy Ltd., Basel, Switzerland, 617-618.

25. Schuster S, Kenanov D (2005) Adenine and adenosine salvage pathways in erythrocytes and the role of S-adenosylhomocysteine hydrolase. A theoretical study using elementary flux modes. The FEBS Journal 272: 5278-5290.

26. Allen DW, Manning N (1994) Abnormal phospholipid metabolism in spur cell anemia: Decreased fatty acid incorporation into phosphatidylethanolamine and increased incorporation into acylcarnitine in spur cell anemia erythrocytes. Blood 84: 1283-1287. 